\title{
Prescription opioid treatment for non-cancer pain and initiation of injection drug use: large retrospective cohort study
}

\author{
James Wilton, ${ }^{1}$ Younathan Abdia, ${ }^{1,2}$ Mei Chong, ${ }^{1}$ Mohammad Ehsanul Karim, ${ }^{2,3}$ Stanley Wong, ${ }^{1}$
} Aaron MacInnes, ${ }^{4,5}$ Rob Balshaw, ${ }^{6}$ Bin Zhao, ${ }^{1}$ Tara Gomes, ${ }^{7,8,9}$ Amanda Yu, ${ }^{1}$ Maria Alvarez, ${ }^{1}$ Richard C Dart, ${ }^{10,11}$ Mel Krajden, ${ }^{1,12}$ Jane A Buxton, ${ }^{1,2}$ Naveed Z Janjua, ${ }^{1,2}$ Roy Purssell ${ }^{1,13}$

For numbered affiliations see end of the article

Correspondence to: J A Buxton jane.buxton@bccdc.ca (ORCID 0000-0003-2295-393X)

Additional material is published online only. To view please visit the journal online.

Cite this as: BMJ 2021;375:e066965 http://dx.doi.org/10.1136/ bmj-2021-066965

Accepted: 25 October 2021

\section{ABSTRACT}

OBJECTIVE

To assess the association between long term prescription opioid treatment medically dispensed

for non-cancer pain and the initiation of injection drug use (IDU) among individuals without a history of substance use.

DESIGN

Retrospective cohort study.

SETTING

Large administrative data source (containing information for about 1.7 million individuals tested for hepatitis ( virus or HIV in British Columbia, Canada) with linkage to administrative health databases, including dispensations from community pharmacies. PARTICIPANTS

Individuals age 11-65 years and without a history of substance use (except alcohol) at baseline.

\section{MAIN OUTCOME MEASURES}

Episodes of prescription opioid use for non-cancer pain were identified based on drugs dispensed between 2000 and 2015. Episodes were classified by the increasing length and intensity of opioid use (acute (lasting $<90$ episode days), episodic (lasting $\geq 90$ episode days; with $<90$ days' drug supply and/ or $<50 \%$ episode intensity), and chronic (lasting $\geq 90$ episode days; with $\geq 90$ days' drug supply and $\geq 50 \%$ episode intensity)). People with a chronic episode were matched 1:1:1:1 on socioeconomic variables to those with episodic or acute episodes and to those who were opioid naive. IDU initiation was

\section{WHAT IS ALREADY KNOWN ON THIS TOPIC}

Several qualitative and observational quantitative studies have assessed the association between prescription opioids and initiation of illicit drug use or injection drug use

Few large longitudinal studies exist, and most studies have instead examined self-reported non-medical prescription opioid use and initiation of heroin use Non-medical use most commonly involves diverted prescription opioids obtained from sources other than a doctor (eg, friends, family, drug dealers); therefore, less is known about medically dispensed opioid treatment for pain

\section{WHAT THIS STUDY ADDS}

In this large longitudinal study of nearly 60000 individuals, objective measures were used for both prescription opioid use and initiation of injection drug use (as opposed to relying on self-report), and medically dispensed opioid treatment for non-cancer pain (as opposed to non-medical use) was assessed

Although the overall rate of injection drug use initiation among individuals with chronic use of prescription opioids was infrequent (3-4\% within five years), the rate was 8-10 times higher than for opioid naive individuals identified by a validated administrative algorithm with high specificity. Cox models weighted by inverse probability of treatment weights assessed the association between opioid use category (chronic, episodic, acute, opioid naive) and IDU initiation.

\section{RESULTS}

59804 participants (14951 people from each opioid use category) were included in the matched cohort, and followed for a median of 5.8 years. 1149 participants initiated IDU. Cumulative probability of IDU initiation at five years was highest for participants with chronic opioid use (4.0\%), followed by those with episodic use (1.3\%) and acute use (0.7\%), and those who were opioid naive $(0.4 \%)$. In the inverse probability of treatment weighted Cox model, risk of IDU initiation was 8.4 times higher for those with chronic opioid use versus those who were opioid naive (95\% confidence interval 6.4 to 10.9$)$. In a sensitivity analysis limited to individuals with a history of chronic pain, cumulative risk for those with chronic use (3.4\% within five years) was lower than the primary results, but the relative risk was not (hazard ratio 9.7 (95\% confidence interval 6.5 to 14.5$)$ ). IDU initiation was more frequent at higher opioid doses and younger ages.

\section{CONCLUSIONS}

The rate of IDU initiation among individuals who received chronic prescription opioid treatment for non-cancer pain was infrequent overall (3-4\% within five years) but about eight times higher than among opioid naive individuals. These findings could have implications for strategies to prevent IDU initiation, but should not be used as a reason to support involuntary tapering or discontinuation of long term prescription opioid treatment.

\section{Introduction}

Injection drug use is associated with substantial morbidity and mortality, ${ }^{1}$ and the prevalence of this practice is increasing in some jurisdictions. ${ }^{23}$ Harms associated with injection drug use include HIV, hepatitis $\mathrm{C}$ virus, infective endocarditis, skin and soft tissue infections, and overdose. ${ }^{45}$ In Canada, the estimated number of people who inject drugs increased from 130000 to 171900 between 2011 and $2016 .{ }^{3}$ In the US, an estimated one million people reported injection drug use in the past 12 months. ${ }^{6}$ Identification of populations at higher risk of initiating injection drug use is critical to prevent transitions to this practice and reduce secondary harms. ${ }^{7}$

Medical use of opioids to treat acute and chronic pain is widespread in North America. ${ }^{48}$ In general, 
prescription opioids are not recommended as first line treatment of chronic non-cancer pain due to limited long term benefit and risk of harms, ${ }^{9-13}$ but adequate non-opioid alternatives are often unavailable or inaccessible (eg, owing to limited insurance coverage, poor availability of multidisciplinary pain clinics, or long wait times for pain management specialists). ${ }^{14}$ Large increases in opioid prescribing in North America began in the late 1990s-driven in part by aggressive marketing and a heightened focus on pain management-and were followed by increases in opioid overdose deaths. ${ }^{15}{ }^{16} \mathrm{~A}$ systematic review of prescription opioid treatment for chronic pain estimated average rates of opioid misuse and addiction to be $21-29 \%$ and $8-12 \%$, respectively. ${ }^{11}$

People prescribed opioids for pain could be at higher risk of initiating illicit or injection drug use. Individuals on long term opioid treatment tend to have a higher prevalence of potential risk factors for illicit or injection drug use, including chronic pain, lower socioeconomic status, mental health issues, and substance use. ${ }^{17-20}$ Further, qualitative studies suggest that prescription opioids can facilitate initiation of heroin or injection drug use, and these transitions can be motivated by increasing tolerance, relief of pain or withdrawal, desire for a better high, or sudden reduced access to opioid drug treatments. ${ }^{21-25}$ Recent initiatives to promote appropriate prescription opioid use have raised concerns related to involuntary tapering or discontinuation and subsequent transitions to the illicit drug market to manage pain or withdrawal. ${ }^{26}$ These concerns are supported by longitudinal studies identifying a heightened risk of harms after discontinuation of opioid treatment. ${ }^{27} 28$

Little is known about the initiation of injection drug use among individuals medically prescribed opioids to manage pain. Instead, most quantitative research has focused on self-reported, so-called nonmedical, prescription opioid use-which most often entails diverted prescription opioids obtained from friends, family members, or drug dealers ${ }^{29}$-and its link to initiation of heroin use. ${ }^{20}$ While self-report is also used to measure the initiation of heroin and injection drug use in these studies, interest in the use of administrative data to identify injection drug use is increasing, because of the wide breadth of these databases and limitations with self-report. ${ }^{6}$ Overall, most administrative studies conducted so far have aimed to quantify prevalence of injection drug use in various jurisdictions (rather than initiation in specific populations, such people who are prescribed opioids for pain). ${ }^{30}$ Assessing initiation is important in order to establish temporality and identify potentially causal risk factors for transitioning to this practice. Identification of injection drug use in administrative data is challenged by the lack of specific diagnostic codes for injection, but validated algorithms exist with varying sensitivity and specificity. ${ }^{30} 31$

In this paper, we used a large administrative data source (of about 1.7 million people) with broad capture of the population in British Columbia, Canada (about a third of the province's population; all individuals tested for HIV or hepatitis C virus), ${ }^{32}$ to examine the association between prescription opioid treatment for non-cancer pain and subsequent initiation of injection drug use among individuals without a history of substance use at baseline.

\section{Methods}

\section{Data sources}

We used the Integrated Data and Evaluative Analytics (IDEAs) cohort (also known as the British Columbia Hepatitis Testers Cohort) to conduct our retrospective cohort study. The databases integrated within IDEAs and their linkage have been described previously (supplementary table A). ${ }^{32}$ The IDEAs cohort is composed of all individuals tested for hepatitis C virus or HIV at the British Columbia Centre for Disease Control Public Health Laboratory between 1992 and 2015, as well as reported cases of hepatitis B and tuberculosis. The laboratory conducts more than 95\% of hepatitis $\mathrm{C}$ virus or HIV diagnostic testing in the province. These data are linked to data on medical visits (1990-2015), hospital admissions (1985-2015), emergency department visits (2012-15), cancer diagnoses (1923-2015), deaths (1985-2015), and pharmacy dispensations (1996-2015).

In British Columbia, HIV or hepatitis $\mathrm{C}$ virus testing is broadly offered to certain populations (eg, pregnant people, baby boomers, sexually active individuals). The IDEAs cohort is estimated to capture about a third of the province's population and $40-50 \%$ of prescription opioid use in the province. ${ }^{18} 32$ Importantly, the IDEAs platform captures historical data on all participants, including data before and after an individual met the inclusion criteria (eg, before and after HIV or hepatitis $\mathrm{C}$ virus testing).

\section{Long term prescription opioid treatment}

Data on opioid medication dispensations were obtained from PharmaNet, a province wide database capturing dispensations from all community pharmacies in British Columbia (1996 onwards), regardless of payer. We identified all episodes of prescription opioid use for non-cancer pain between 1996 and $2015 .{ }^{33}$ As defined by others, an episode starts with an incident opioid prescription (defined as no drug supply available in the past six months) and ends once there has been another six months with no drug supply. ${ }^{33}$ When creating these episodes, we did not consider low dose codeine formulations (<30 mg per tablet), opioid formulations primarily used for cough suppression or opioid agonist treatment, injectable opioid formulations, or opioid dispensations occurring after a cancer or palliative care record. $^{1834}$

Based on episode length, we initially characterised each episode as either acute (lasting < 90 episode days) or long term ( $\geq 90$ episode days). ${ }^{33}$ Long term episodes were further categorised as episodic or chronic, based on days' of drug supply (that is, the number of calendar days within an episode covered by drug supply) and episode intensity (that is, the percentage 
of episode days covered by drug supply). Episodic episodes contained fewer than 90 days' drug supply and/or less than $50 \%$ of episode intensity, and chronic episodes contained at least 90 days' drug supply and at least 50\% episode intensity. ${ }^{1834}$ We have extensively characterised these definitions in a previous analysis. ${ }^{18}$

\section{Initiation of injection drug use}

Participants were considered to have initiated injection drug use when the following two criteria occurred within one year of each other:

- Evidence of a problem related to the use of potentially injectable drugs (eg, diagnostic codes for dependence to opioids, cocaine, amphetamines, or benzodiazepines; prescription of opioid agonist treatment; supplementary table B); and

- Diagnosis of a possible injection related infection (eg, endocarditis, bacteraemia or sepsis, skin or soft tissue infections; supplementary table B).

In a validation study using the IDEAs cohort and linked surveillance data with self-reported injection drug use, this algorithm had a sensitivity of $63 \%$ and specificity of $90 \%$ for identifying people who inject drugs. ${ }^{30}$ Despite the availability of alternative algorithms with a better balance between sensitivity and specificity, ${ }^{30}$ we opted for a highly specific algorithm that included a requirement for an injection related infection, because drug related diagnostic codes do not specify the route of drug administration (eg, injection $v$ oral) and-given our study population-these codes could be flagging dependence to prescription opioids taken orally.

\section{Study design (matched cohort)}

We created a matched cohort to examine the association between long term prescription opioid treatment and initiation of injection drug use. Each matched grouping included one person with a chronic episode of opioid use, one person with episodic opioid use, one person with acute opioid use, and an opioid naive person-all who entered the analysis in the same year and at about the same age (within a difference of 1 year of age). In this article, we refer to these categories within each matched group as the prescription opioid use categories (chronic, episodic, acute, opioid naïve).

To create the matched cohort, individuals with long term opioid use were classified into two groups, according to whether their first long term episode between 2000 and 2015 was episodic or chronic. Individuals with chronic opioid use were matched 1:1 to those with episodic opioid use, based on sociodemographic variables (birth year, sex, ethnic origin) and year of index date. Index date was defined as the initiation date of long term use (that is, start date of the first long term episode plus 90 days).

We next matched individuals with chronic opioid use (based on birth year, sex, and ethnic origin) to two groups of individuals without a history of long term prescription opioid use at baseline (1:1:1 to opioid naive and acute use individuals). These two groups of individuals without long term prescription opioid use were assigned the index date (baseline) of the person with chronic opioid use to whom they were matched. ${ }^{35}$ The two groups were defined on the basis of prescription opioid use before their index date (that is, no history of opioid use for opioid naive, and $\geq 1$ acute episode for acute use).

\section{Eligibility criteria (matched cohort)}

We applied several inclusion and exclusion criteria before and during the matching process. We included individuals aged between 11 and 65 years at the index date (the injection drug use algorithm was only validated for this age range $)^{30}$ and who had a record of living in British Columbia at least five years before index date.

We excluded individuals who had a cancer or palliative care diagnosis or evidence of substance use problems before index date. We were conservative with regards to the second exclusion criteria, and excluded all individuals meeting a more sensitive version of our injection drug use algorithm (sensitivity $91 \%$, specificity $72 \%$; supplementary table B). ${ }^{30}$ This algorithm excluded the injection related infection requirement and captured a broader range of substance use diagnostic codes (but did not include alcohol). To further improve sensitivity, we also excluded individuals who were living with HIV or had been given a diagnosis of hepatitis $\mathrm{C}$ virus.

Before matching, we also excluded individuals who had missing sex or high level geographical information (health authority). However, we included individuals who were missing social or material deprivation information, because the lack of geographical information could be a proxy for homelessness.

\section{Statistical analysis}

Participants were followed from the index date to the earliest of the following: injection drug use initiation, 66 years of age, out-migration (two years after the last record in linked databases), initiation of a long term prescription opioid episode (only applicable to individuals in the acute use and opioid naive categories), study end (end of 2015 or 10 years of follow-up), or death. Individuals in the opioid naive and episodic use categories were not censored at initiation of an acute or chronic episode, respectively.

We used cumulative incidence curves to measure the cumulative probability of injection drug use initiation and Cox models to quantify the relative association between opioid use category (chronic, episodic, acute, opioid naive) and injection drug use initiation. In subanalyses, we stratified individuals with chronic opioid use by average daily dose across their first chronic episode. Average daily dose was calculated as the cumulative morphine equivalents prescribed during the episode divided by number of episode days covered by drug supply.

We weighted Cox models by inverse probability of treatment weights (IPTW). ${ }^{36}$ The generalised propensity 
scores used to derive IPTW were calculated from a multinomial logistic regression model with opioid use category as the outcome and sex, age, ethnic origin, health authority, material deprivation, major mental illness, alcohol use problem, and chronic pain as independent variables (all measured at the index date; supplementary table B). Weights were truncated at 1st and 99th centiles. ${ }^{36}$ Models used a robust sandwich covariate matrix to account for intracluster correlation within each matched group. ${ }^{37}$ We used the cumulative sums of Martingale residuals and the Kolmogorov type supremum test to assess the non-proportional hazards assumption. $^{38}$

\section{Sensitivity analyses}

We conducted several sensitivity analyses, including a model with death as a competing risk, ${ }^{39}$ a model with inverse probability of censoring weights to account for artificial censoring at initiation of a long term episode (only applicable to individuals in the acute use and opioid naive categories), ${ }^{40}$ and a model using an alternate matched cohort limited to individuals with a history of chronic pain. ${ }^{4142}$

\section{Patient and public involvement}

This cohort study was developed without patient involvement owing to the retrospective, administrative, and de-identified nature of the data sources. The study was developed in response to concerns expressed by patients and the public regarding the contribution of prescription opioids to the illicit overdose crisis in British Columbia. These concerns were brought forward to members of the research team through peer based organisations of people with lived and living experience of substance use, the media, concerned friends and family members, providers, and provincial committees involved in the overdose response.

\section{Results}

Study population

The IDEAs PharmaNet database includes information for about 1.7 million individuals (1996-2015). Of 159109 eligible individuals who had a first long term prescription opioid episode between 2000 and 2015 (fig 1), 14975 had a first long term episode that was chronic (that is, lasting $\geq 90$ episode days; with at least 90 days' drug supply and at least 50\% episode intensity). Almost all eligible people with a chronic episode (apart from 24 (0.2\%) individuals) were matched $(1: 1: 1: 1)$ to an individual in the other opioid use categories (episodic, acute, opioid naive). The final matched cohort included 59804 people (14951 in each category).

\section{Characteristics of study participants and success of IPTW weighting}

Overall, 30140 (50.4\%) participants were male and the median age was 44 (interquartile range 35-55) years. The footnotes of table 1 show the breakdown of matched sociodemographic variables. With increasing length and intensity of prescription opioid use (that is, from opioid naive to chronic use), we observed an increasing proportion of people who were more materially deprived or had history of major mental illness, alcohol use problems, or chronic pain (table 1). Standardised mean differences were 0.1 or more for all covariates before weighting by IPTW (except for matched variables) and all were less than 0.1 after weighting (supplementary table $\mathrm{C}$ ).

\section{Prescription opioid episode characteristics}

Half of the chronic opioid episodes were longer than two years, contained more than 1.5 years of drug supply, and had an average daily dose of higher than 32 morphine equivalents (supplementary table D). The corresponding characteristics were lower for episodic episodes (171 days, 22 days, and 22 morphine equivalents, respectively). Supplementary table D contains additional information on episode characteristics (eg, inferred indication, most common opioid formulation). Our previous analysis showed that most acute episodes (about 70\%) contain $\leq 7$ days' supply of opioids. ${ }^{18}$

\section{Study follow-up and injection drug use initiation}

The 59804 individuals in the matched cohort were followed for a median of 5.8 years (interquartile range 3.0-9.4), and 1149 participants initiated injection drug use (table 2). Risk of initiation was highest for the chronic use category, followed by the episodic use, acute use, and opioid naive categories (table 2 , fig 2). In the chronic use category, risk of initiation was higher at younger ages (table 2, fig 2) and greater average daily dose (table 2, fig 2). Among individuals with chronic use who initiated injection drug use, the median time to identification of injection drug use through the administrative algorithm was 3.6 years (interquartile range 1.7-6.0).

\section{Cox models}

The magnitude of association between opioid use category ( $v$ opioid naive individuals) and injection drug use initiation increased with greater intensity and length of use (that is, chronic use had a greater association than episodic use, which was in turn greater than acute use; table 3). In the IPTW Cox model, risk of injection drug use initiation was 8.4 times (95\% confidence interval 6.4 to 10.9) higher for people in the chronic opioid use category than for opioid naive individuals, 2.7 times (2.1 to 3.6) higher for people in the episodic use category, and 1.4 times (1.0 to 2.0) higher for people in the acute use category. Table 4 shows other characteristics associated with initiation of injection drug use.

\section{Sensitivity analyses}

Sensitivity analyses were generally consistent with our primary results (supplementary table E). However, limiting the analysis to individuals with a history of chronic pain lowered the cumulative probabilities of injection drug use initiation but did not lower the hazard ratios (table 3). 


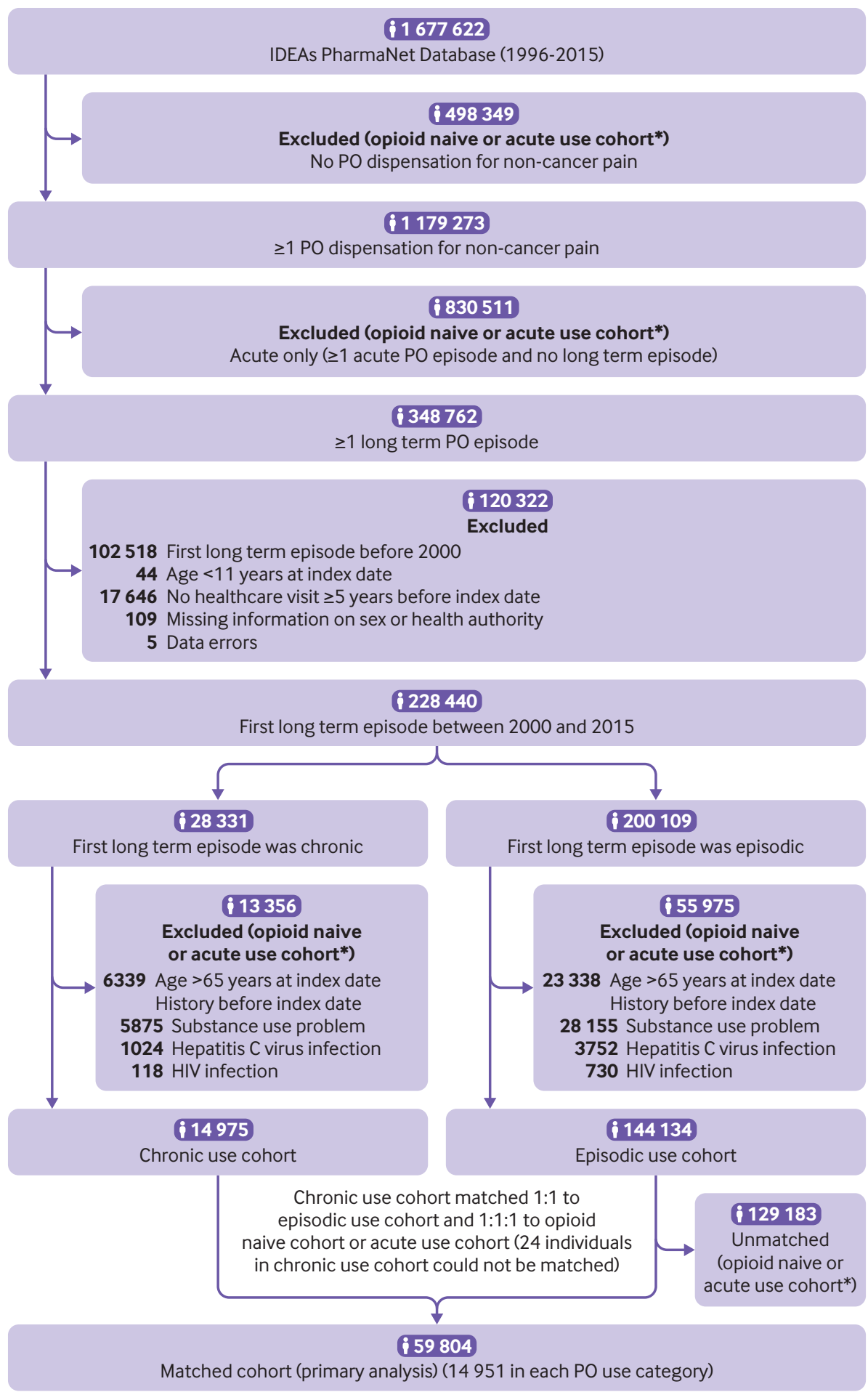

Fig 1 | Study flowchart. IDEAs=Integrated Data and Evaluative Analytics data platform; PO=prescription opioid. *Opioid naive or acute use cohort contains the pool of potential opioid naive or acute use individuals available for matching to the chronic use category; see methods for more detailed definitions of opioid use episodes

\section{Discussion}

\section{Principal findings}

In this longitudinal analysis of roughly 60000 matched individuals selected from a large administrative data source (all individuals tested for HIV or hepatitis C virus in the Canadian province of British Columbia), the rate of injection drug use initiation among individuals who received chronic prescription opioid treatment for non- cancer pain was infrequent overall (3-4\% within five years), but 8-10 times higher than among opioid naive individuals. Notably, of the almost 15000 individuals on chronic opioid treatment, half had used opioid treatments on most days for more than two years, and initiation of injection drug use was more frequent at higher doses and younger ages. We excluded people older than 65 years or with a history of substance use 


\begin{tabular}{|c|c|c|c|c|}
\hline & $\begin{array}{l}\text { Chronic use } \\
(n=14951)\end{array}$ & $\begin{array}{l}\text { Episodic use } \\
(n=14951)\end{array}$ & $\begin{array}{l}\text { Acute use } \\
(n=14951)\end{array}$ & $\begin{array}{l}\text { Opioid naive } \\
(n=14951)\end{array}$ \\
\hline Matched variables (sex, birth year, year of index date, approximate age ( \pm 1 year), ethnic origin) & \multicolumn{4}{|c|}{ Approximately the same across categories* } \\
\hline \multicolumn{5}{|l|}{ Health authority (British Columbia) } \\
\hline Vancouver Coastal & 2931 (19.6) & $3230(21.6)$ & $4087(27.3)$ & $4843(32.4)$ \\
\hline Fraser & $4904(32.8)$ & $5220(34.9)$ & $5228(35.0)$ & 4998 (33.4) \\
\hline Vancouver Island & $2666(17.8)$ & 2549 (17.1) & $2366(15.8)$ & 2095 (14.0) \\
\hline Interior & $3216(21.5)$ & $2564(17.2)$ & $2216(14.8)$ & $2130(14.3)$ \\
\hline Northern & $1234(8.3)$ & $1388(9.3)$ & $1054(7.1)$ & $885(5.9)$ \\
\hline \multicolumn{5}{|l|}{ Urbanicity } \\
\hline Urban & $12644(84.6)$ & $12696(84.9)$ & $12999(86.9)$ & $13201(88.3)$ \\
\hline Rural & $2254(15.1)$ & $2186(14.6)$ & $1904(12.7)$ & 1694 (11.3) \\
\hline Missing & $53(0.4)$ & $69(0.5)$ & $48(0.3)$ & $56(0.4)$ \\
\hline \multicolumn{5}{|l|}{ Material deprivationt } \\
\hline 1 (least) & $2405(16.1)$ & $2873(19.2)$ & $3444(23.0)$ & $3678(24.6)$ \\
\hline 5 (most) & $3295(22.0)$ & 2969 (19.9) & $2439(16.3)$ & $2394(16.0)$ \\
\hline Missing & $97(0.7)$ & $87(0.6)$ & $67(0.5)$ & $72(0.5)$ \\
\hline \multicolumn{5}{|l|}{ Social deprivationt } \\
\hline 1 (least) & $2222(14.9)$ & $2496(16.7)$ & $2648(17.7)$ & $2671(17.9)$ \\
\hline 5 (most) & $4044(27.1)$ & $3622(24.2)$ & $3481(23.3)$ & $3658(24.5)$ \\
\hline Missing & $97(0.7)$ & $87(0.6)$ & $67(0.5)$ & $72(0.5)$ \\
\hline Chronic pain & $8452(56.5)$ & $6905(46.2)$ & $5232(35.0)$ & $3197(21.4)$ \\
\hline Major mental illness & $2937(19.6)$ & $2338(15.6)$ & $1837(12.3)$ & $1362(9.1)$ \\
\hline Alcohol use problem & $996(6.7)$ & $686(4.6)$ & $409(2.7)$ & $255(1.7)$ \\
\hline \multicolumn{5}{|l|}{ Elixhauser comorbidity indext } \\
\hline 0 & $7171(48.0)$ & $8793(58.8)$ & $10206(68.3)$ & $11082(74.1)$ \\
\hline$\geq 4$ & 2234 (14.9) & $1341(9.0)$ & $838(5.6)$ & $649(4.3)$ \\
\hline \multicolumn{5}{|c|}{ 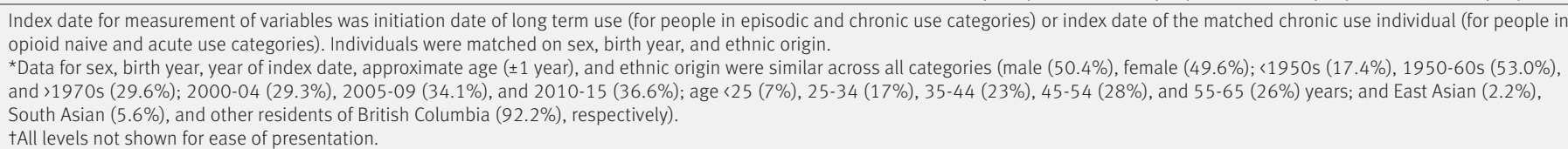 } \\
\hline
\end{tabular}

problem (except alcohol) at baseline. Aspects unique to our analysis include the use of a population based, dispensation data source to identify opioid treatment (all prescriptions dispensed from community pharmacies, regardless of payer) and the application of a highly specific administrative algorithm (validated in our data source) to identify initiation of injection drug use. ${ }^{18} 30$ Our analysis fills an important gap in the literature, which has primarily focused on self-reported non-medical prescription opioid use (NMPOU) and heroin use, ${ }^{20}$ and also contributes to a relatively new field of research using administrative algorithms to identify injection drug use. Importantly, we were unable to determine the underlying reasons for initiation of injection drug use, or whether these initiations were causally related to opioid treatment.

\begin{tabular}{|c|c|c|c|c|c|c|}
\hline \multirow[b]{2}{*}{ Category } & \multirow[b]{2}{*}{$\begin{array}{l}\text { No of } \\
\text { participants }\end{array}$} & \multicolumn{2}{|c|}{ Follow-up (person years) } & \multirow[b]{2}{*}{$\begin{array}{l}\text { No of incident } \\
\text { IDU initiations }\end{array}$} & \multirow{2}{*}{$\begin{array}{l}\text { Rate of IDU } \\
\text { initiation } \\
\text { (per } 1000 \\
\text { person years) }\end{array}$} & \multirow{2}{*}{$\begin{array}{l}5 \text { year cumulative } \\
\text { probability of IDU } \\
\text { initiation (\%) }\end{array}$} \\
\hline & & Total & $\begin{array}{l}\text { Median } \\
\text { (interquartile range) }\end{array}$ & & & \\
\hline Full matched cohort* & 59804 & 350131 & $5.8(3.0-9.4)$ & 1149 & 3.3 & 1.6 \\
\hline Opioid naive & 14951 & 87939 & $5.8(3.1-9.4)$ & 73 & 0.8 & 0.4 \\
\hline Acute & 14951 & 77878 & $4.9(2.4-8.1)$ & 98 & 1.3 & 0.7 \\
\hline Long term (all) & 29902 & 194314 & $6.3(3.4-10.0)$ & 978 & 5.0 & 2.6 \\
\hline Episodic & 14951 & 93780 & $6.5(3.5-10.0)$ & 248 & 2.6 & 1.3 \\
\hline Chronic & 14951 & 90534 & $6.2(3.2-9.8)$ & 730 & 8.1 & 4.0 \\
\hline \multicolumn{7}{|c|}{ Average daily dose (chronic use only; morphine equivalents)† } \\
\hline$<50$ & 9887 & 58499 & $5.9(3.0-9.7)$ & 291 & 5.0 & 2.4 \\
\hline $50-89$ & 2574 & 15259 & $6.0(3.3-9.0)$ & 139 & 9.1 & 4.7 \\
\hline 90-199 & 1695 & 11187 & $7.1(4.0-10.0)$ & 175 & 15.6 & 7.9 \\
\hline$\geq 200$ & 795 & 5589 & $7.9(4.6-10.0)$ & 125 & 22.4 & 11.0 \\
\hline \multicolumn{7}{|c|}{ Age (chronic use only; years) $\dagger$} \\
\hline$<25$ & 1083 & 6953 & $6.6(3.8-10.0)$ & 129 & 18.6 & 9.4 \\
\hline $25-34$ & 2502 & 15703 & $6.4(3.7-10.0)$ & 209 & 13.3 & 6.6 \\
\hline $35-44$ & 3382 & 23540 & $7.8(4.4-10.0)$ & 193 & 8.2 & 4.0 \\
\hline $45-65$ & 7984 & 44338 & $5.4(2.7-8.7)$ & 199 & 4.5 & 2.3 \\
\hline
\end{tabular}



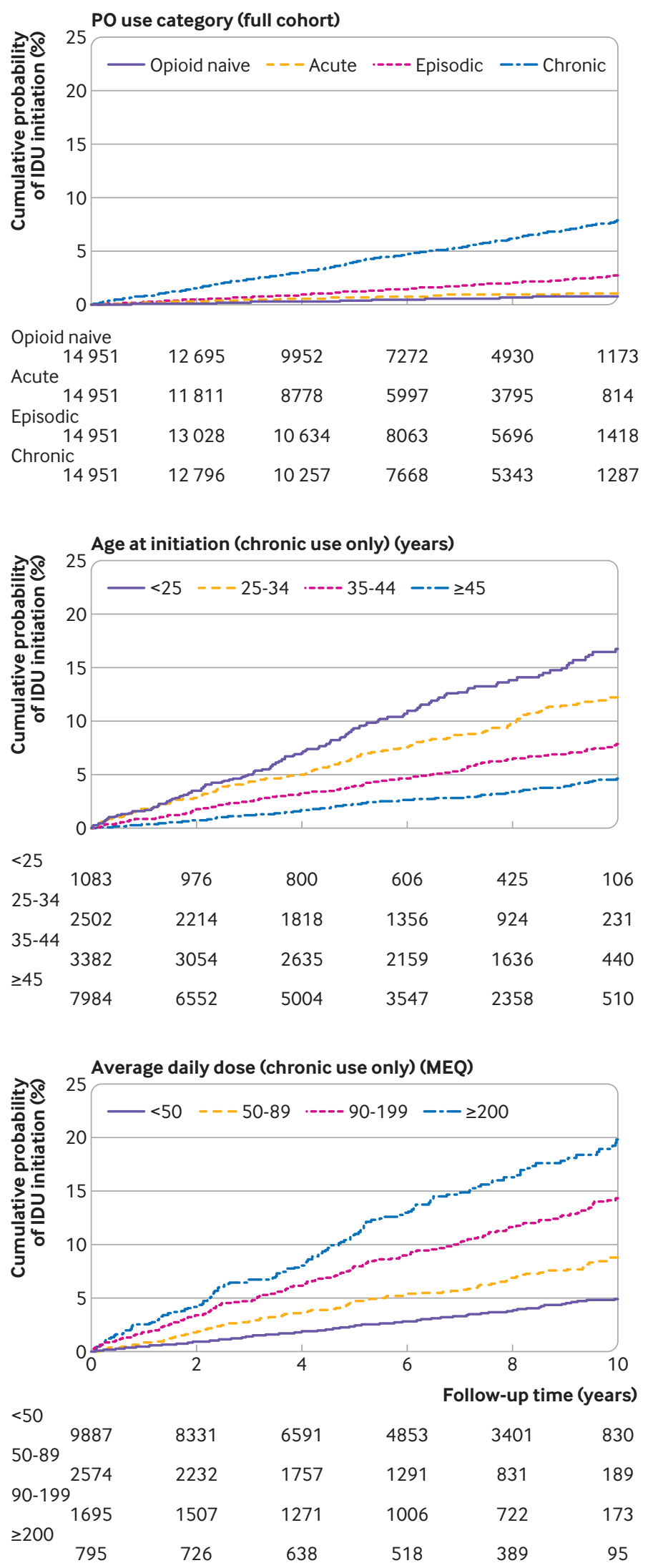

Fig 2 | Cumulative incidence of injection drug use initiation by prescription opioid use category, age at initiation of chronic episode (chronic use category only), and average daily dose of chronic episode (chronic use category only). $\mathrm{PO}=$ prescription opioid; $M E Q=$ morphine equivalents; $I D U=i n j e c t i o n$ drug use. See methods for more details on opioid use categories. Average daily dose calculated as the cumulative morphine equivalents dispensed during episode divided by number of episode days covered by drug supply 


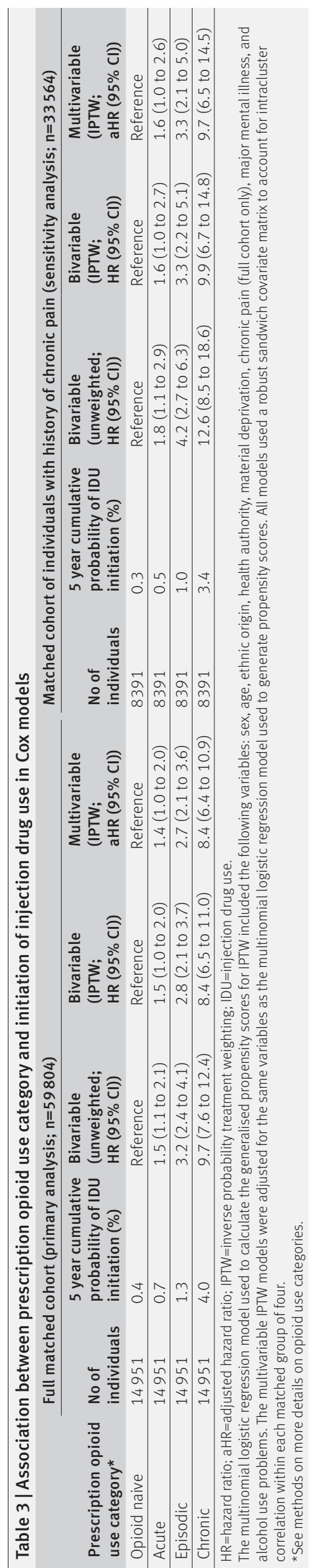

Implications for clinicians and policy makers

Our findings could have implications for strategies to prevent the initiation of injection drug use (eg, more judicious opioid prescribing), but should not be used as a reason to support involuntary tapering or discontinuation of prescription opioid treatment. Abrupt discontinuation could lead to some individuals to access the increasingly dangerous illicit drug market, ${ }^{23272843}$ and could explain some of the initiations of injection drug use in our analysis. Inadequately managed pain and denial of pain drug treatments have been identified as risk factors for illicit drug use, particularly among people with history of substance use. ${ }^{29} 4344$ The Canadian Pain Task Force has identified a need to foster shared decision making between providers and individuals living with chronic pain and "support opioid prescribing that balances the benefits and harms of these medications based on the needs of the individual." 45 Improved access to multidisciplinary approaches to treat pain is also needed. $^{14} 45$ Regarding non-opioid interventions, evidence suggests that some non-invasive (eg, exercise therapy, multidisciplinary rehabilitation, cognitive behavioural therapy) and invasive (eg, nerve blocks) treatments can improve function and/or reduce pain for specific conditions. ${ }^{4647}$

The dose-response relation suggests that interventions aimed at preventing and treating prescription opioid dependence, appropriately tapering or discontinuing opioid use, and adequately treating pain (because dose might be a proxy for pain severity) could reduce the risk of injection drug use initiation. Guidelines generally recommend the avoidance of escalation to high doses, slow and voluntary tapering to the lowest effective dose (and potentially discontinuation) for people receiving high doses, and a multidisciplinary programme for individuals experiencing challenges in tapering. ${ }^{12} 13$ Best practices for treating opioid dependence include provision of opioid agonist treatment in conjunction with psychosocial interventions and supports. Harm reduction interventions (eg, sterile drug use equipment, safer injection sites, and use of prescription opioids as a pharmaceutical alternative to illicit drugs) could reduce the risk of downstream harms from illicit or injection drug use.

We also found male sex, younger age, higher material deprivation, and history of mental illness and alcohol use problems to be associated with the initiation of injection drug use. These populations might also benefit from enhanced efforts to prevent initiation of injection.

\section{Comparison to other findings}

Our large longitudinal analysis supports findings from several surveys and smaller longitudinal studies demonstrating an association between self-reported NMPOU and initiation of heroin use. ${ }^{20}$ While many of these studies enrolled specific populations and might not be widely generalisable, two large analyses of pooled data from a nationally representative survey in 


\begin{tabular}{|c|c|}
\hline & Adjusted hazard ratio $(95 \% \mathrm{Cl})$ \\
\hline \multicolumn{2}{|c|}{ Prescription opioid use category ( $v$ opioid naive) ${ }^{*}$} \\
\hline Acute & $1.4(1.0$ to 2.0$)$ \\
\hline Episodic & $2.7(2.1$ to 3.6$)$ \\
\hline Chronic & $8.4(6.4$ to 10.9$)$ \\
\hline Male sex ( $v$ female) & $1.6(1.4$ to 1.8$)$ \\
\hline \multicolumn{2}{|l|}{ Age ( $v 55-65$ years) } \\
\hline$<25$ & $4.8(3.7$ to 6.2$)$ \\
\hline $25-34$ & $3.3(2.6$ to 4.2$)$ \\
\hline $35-44$ & $2.0(1.6$ to 2.6$)$ \\
\hline $45-54$ & $1.1(0.9$ to 1.5$)$ \\
\hline \multicolumn{2}{|c|}{ Ethnic origin ( $v$ other residents of British Columbia) } \\
\hline South Asian & $0.5(0.3$ to 0.7$)$ \\
\hline East Asian & $0.4(0.2$ to 0.9$)$ \\
\hline \multicolumn{2}{|c|}{ Material deprivation ( $v 1$ (least)) } \\
\hline 2 & $1.3(1.0$ to 1.6$)$ \\
\hline 3 & $1.4(1.1$ to 1.8$)$ \\
\hline 4 & $1.4(1.1$ to 1.8$)$ \\
\hline 5 (most) & $1.6(1.3$ to 2.0$)$ \\
\hline Missingt & $2.1(1.2$ to 3.7$)$ \\
\hline Chronic pain & $0.8(0.7$ to 0.9$)$ \\
\hline Alcohol use problem & $2.7(2.2$ to 3.3$)$ \\
\hline Major mental illness & $1.6(1.3$ to 1.8$)$ \\
\hline \multicolumn{2}{|c|}{$\begin{array}{l}\text { IPTW=inverse probability of treatment weighting. } \\
\text { Model also adjusted for health authority (no statistically significant associations). The multinomial logistic } \\
\text { regression model used to calculate the generalised propensity scores for IPTW included all the variables } \\
\text { included in the multivariable Cox model. The Cox model used a robust sandwich covariate matrix to account for } \\
\text { intracluster correlation within each matched group. } \\
\text { *See methods on more details on opioid use categories. } \\
\text { †Missing material deprivation was retained in the model because it could be a proxy for homelessness. }\end{array}$} \\
\hline
\end{tabular}

the US (National Survey on Drug Use and Health) found that incidence of heroin use initiation was 19 times higher among participants reporting previous NMPOU (unadjusted analysis), ${ }^{48}$ and that young individuals (aged 12-21 years) with a history of NMPOU were at 13 times higher risk of initiating heroin use. ${ }^{49}$ In one of the only studies to assess medically dispensed opioids for pain, high dose treatment was associated with a 2.5-fold higher risk of heroin use among military veterans in the US with a high prevalence of HIV (51\%) and hepatitis C virus (33\%). ${ }^{50}$

Our overall five year cumulative rate of injection drug use initiation after chronic prescription opioid use (3.4$4.0 \%$ ) is similar to the widely cited transition rate from NMPOU to heroin use (3.6\% within five years) from the National Survey on Drug Use and Health. ${ }^{48}$ While any comparisons require caution, a common motivation for NMPOU is the relief of pain, ${ }^{29}$ perhaps explaining the similarity. Higher rates of transition to heroin use have been observed in studies of specific populations. ${ }^{51-54}$ The overall rate of injection drug use initiation in our analysis is also supported by estimated rates of misuse and addiction after prescription opioid treatment for chronic pain $\left(21-29 \%\right.$ and $8-12 \%$, respectively), ${ }^{11}$ because only a proportion of misuse or addiction would be expected to progress to injection. Initiation rates in our analysis were subject to several potential sources of over-estimation (eg, study limited to individuals tested for HIV or hepatitis C virus, exclusion of individuals older than 65 years of age, possible inclusion of people accessing prescription opioids for non-pain purposes, potential low positive predictive value) and underestimation (eg, low sensitivity of the injection drug use algorithm, exclusion of people with a history of substance use), and should therefore be interpreted with caution.

\section{Strengths and weaknesses of the study}

Confounding could partly explain the association between prescription opioids and initiation of injection drug use, and could limit the extent to which firm conclusions can be drawn. We noted an increasing prevalence of potential risk factors for injection drug use across the gradient of increasing length and intensity of prescription opioid use (that is, from opioid naive to chronic use), including chronic pain, higher social or material deprivation, major mental illness, and alcohol use problems. To adjust for this potential source of bias, we matched on sociodemographic variables, used IPTW to balance further differences, and performed a sensitivity analysis limited to individuals with chronic pain (a key confounder by indication because individuals might transition to injection drug use to manage uncontrolled chronic pain, independent of their current or previous prescription opioid use). Among people with chronic opioid use in this sensitivity analysis, the cumulative probability of initiation decreased but the relative strength of association (hazard ratio) did not. Possible sources of residual confounding include the use of administrative databases to identify confounders (a source of misclassification bias), potential inclusion of individuals accessing prescription opioids for nonpain purposes (eg, euphoric effects, dependence, offlabel opioid agonist treatment), and the inability to adjust for severity of pain.

Several strengths and weaknesses were related to the use of our administrative algorithm to identify injection drug use. Strengths include the high specificity of the algorithm, advantages compared to the use of selfreport, and contribution to a relatively new field of research exploring the use of such algorithms (which is inherently challenged by the lack of diagnostic codes specific to injection drug use). Limitations include low sensitivity and low potential positive predictive value (owing to expected low prevalence of the outcome), validation of our algorithm via self-report (although self-report is generally considered the gold standard), inability to identify exact date of injection initiation, and delays between actual initiation and algorithm identification.

Our analysis had several other limitations, including challenges using pharmacy dispensation data to determine how drug treatments were actually taken and possible inclusion of people with undetected injection drug use at baseline (owing to the imperfect sensitivity of the algorithm). Furthermore, the doseresponse analysis was based on the average daily dose across the full chronic episode and could include dispensations after initiation of injection drug use. We did not compare prescription opioids to alternative pain treatments, and this requires further research. Also, our analysis did not take into account the time varying nature of potential confounders; instead, covariates 
were measured as binary variables at study start. Although the study end date was 2015, our analysis captures an important period in British Columbia when prescription opioid use increased and could have contributed to the illicit opioid overdose epidemic that started in 2015-16 (by facilitating transitions to illicit or injection drug use). Finally, our study population was primarily limited to people tested for HIV or hepatitis $\mathrm{C}$ virus, potentially limiting generalisability of study findings. In particular, the absolute rates of injection drug use initiation could be higher in our study sample than the general population. However, HIV and hepatitis C virus testing is broadly offered to some populations in British Columbia (eg, pregnant people, baby boomers, sexually active individuals).

\section{Conclusion}

The rate of injection drug use initiation among individuals who received chronic prescription opioid treatment for non-cancer pain was infrequent overall (3-4\% within five years), but 8-10 times higher than opioid naive individuals. Rate of initiation was more frequent with greater doses and at younger ages. Our findings could help inform strategies and policies to prevent further transitions to injection drug use and related harms secondary to such use, but should not be used as a reason to inappropriately taper or discontinue long term prescription opioid treatment.

\section{AUTHOR AFFILIATIONS}

${ }^{1}$ British Columbia Centre for Disease Control, Vancouver, BC, Canada

${ }^{2}$ School of Population and Public Health, University of British Columbia, Vancouver, BC, Canada

${ }^{3}$ Centre for Health Evaluation and Outcome Sciences, St Paul's Hospital Vancouver, BC, Canada

${ }^{4}$ Pain Management Clinic, JPOCSC, Fraser Health Authority, Surrey, BC, Canada

${ }^{5}$ Department of Anaesthesiology, Pharmacology and Therapeutics, Faculty of Medicine, University of British Columbia, Vancouver, BC, Canada

${ }^{6}$ George and Fay Yee Centre for Healthcare Innovation, University of Manitoba, Winnipeg, MB, Canada

${ }^{7}$ Leslie Dan Faculty of Pharmacy, University of Toronto, Toronto, ON, Canada

${ }^{8}$ Li Ka Shing Knowledge Institute, St Michael's Hospital, Toronto, ON, Canada

${ }^{9}$ ICES, Toronto, ON, Canada

${ }^{10}$ Rocky Mountain Poison and Drug Safety, Denver Health and Hospital Authority, Denver, CO, USA

${ }^{11}$ Department of Emergency Medicine, University of Colorado Health Sciences Center, Denver, CO, USA

${ }^{12}$ Department of Pathology and Laboratory Medicine, University of British Columbia, Vancouver, BC, Canada

${ }^{13}$ Department of Emergency Medicine, University of British Columbia, Vancouver, BC, Canada

We thank the Provincial Health Services Authority, British Columbia Ministry of Health, and British Columbia Cancer staff for their assistance in data access, procurement, and management; and the residents of British Columbia, whose data are integrated in IDEAs and the British Columbia Hepatitis Testers Cohort. This work was carried out on the unceded traditional and ancestral territories of the Coast Salish people. All inferences, opinions, and conclusions drawn in this manuscript are those of the authors, and do not reflect the opinions or policies of the data stewards.

Contributors: JAB, RP, and NZJ conceptualised the study. JAB, RP, and NZJ (primary investigators) and TG, RCD, and RB (co-investigators) developed the grant to acquire funding. Project administration and supervision was provided by JAB, RP, NZJ, and MA. JAB, RP, NZJ, JW, and YA developed the study design; MEK, RB, and BZ contributed to the methodology. MA, SW, and AY were involved in data linkage and data management. MC and YA created the analytical dataset with assistance from SW and AY. JW and YA performed the formal analysis, and RP and JW wrote the original draft of the manuscript. JW and YA had full access to data in the study. JW accepts full responsibility for the work and the conduct of the study and is the guarantor. All authors reviewed the manuscript and contributed to interpretation of the data. The corresponding author attests that all listed authors meet authorship criteria and that no others meeting the criteria have been omitted.

Funding: This work was supported by British Columbia Centre for Disease Control and the Canadian Institutes of Health Research (PJT-156117, NHC-142832). The funders were not involved in the study design; in the collection, analysis, and interpretation of data; in the writing of the report; or in the decision to submit the article for publication.

Competing interests: All authors have completed the ICMJE uniform disclosure form at www.icmje.org/disclosure-of-interest/ and declare: support from British Columbia Centre for Disease Control and the Canadian Institutes of Health Research for the submitted work; outside of the submitted work, MK has received research grant funding via his institution from Roche Molecular Systems, Siemens Healthcare Diagnostics, and Hologic; MEK has received consulting fees from Biogen; and RCD has performed research and consulted on the over-the-counter product Tylenol for the consumer division of Johnson \& Johnson. RCD is executive director of the RADARS System, which performs post-marketing surveillance of prescription drugs. This information is primarily used for regulatory purposes. Subscribers to the RADARS System include the US Food and Drug Administration and some manufacturers of prescription analgesics or treatment for opioid addiction. These companies include Collegium, Purdue Pharma, and Indivior. All other authors report no financial relationships with any organisations that might have an interest in the submitted work in the previous three years. All authors report no other relationships or activities that could appear to have influenced the submitted work. Ethical approval: Data linkage to establish IDEAs and the British Columbia Hepatitis Testers Cohort was performed under the British Columbia Centre for Disease Control's public health mandate. This study was approved by the University of British Columbia's research ethics board (H14-01649).

Data sharing: The IDEAs cohort was made possible through a multi-agency collaboration, including the British Columbia Centre for Disease Control, British Columbia Centre for Disease Control's Public Health Laboratory, British Columbia Cancer, and the British Columbia Ministry of Health. The integrated dataset involved the linkage of de-identified case, laboratory testing, and cancer data with health services administrative data. Authorised users have access to de-identified data based on roles and projects. Access to the deidentified data is not permitted outside of Canada. Use of the data is limited to the objectives described in the Information Sharing Plan. Individuals interested in becoming external collaborators can contact Naveed Janjua (naveed.janjua@bccdc.ca). The British Columbia Centre for Disease Control requires all internal and external researchers with access to analytical datasets to complete annual or more frequent privacy or confidentiality and information security and awareness training, sign confidentiality forms, and complete a concept note and data request.

The lead authors (JW, RP, NZJ, JAB) affirm that the manuscript is an honest, accurate, and transparent account of the study being reported; that no important aspects of the study have been omitted; and that any discrepancies from the study as originally planned have been explained.

Dissemination to participants and related patient and public communities: Results will be presented to peer based organisations of people with lived and living experience of substance use and provincial committees involved in the overdose response. We also plan to disseminate results more broadly through IDEAs and the British Columbia Hepatitis Testers Cohort Twitter page (@BCHepCo) and website (https://bchtc.med.ubc.ca/).

Provenance and peer review: Not commissioned; externally peer reviewed.

This is an Open Access article distributed in accordance with the Creative Commons Attribution Non Commercial (CC BY-NC 4.0) license, which permits others to distribute, remix, adapt, build upon this work non-commercially, and license their derivative works on different terms, provided the original work is properly cited and the use is noncommercial. See: http://creativecommons.org/licenses/by-nc/4.0/. 
1 Mathers BM, Degenhardt L, Bucello C, Lemon J, Wiessing L, Hickman M. Mortality among people who inject drugs: a systematic review and meta-analysis. Bull World Health Organ 2013;91:102-23. doi:10.2471/BLT.12.108282

2 Zibbell JE, Asher AK, Patel RC, et al. Increases in Acute Hepatitis C Virus Infection Related to a Growing Opioid Epidemic and Associated Injection Drug Use, United States, 2004 to 2014. Am J Public Health 2018:108:175-81. doi:10.2105/AJPH.2017.304132

3 Jacka B, Larney S, Degenhardt L, et al. Prevalence of Injecting Drug Use and Coverage of Interventions to Prevent HIV and Hepatitis C Virus Infection Among People Who Inject Drugs in Canada. Am I Public Health 2020;110:45-50. doi:10.2105/AJPH.2019.305379

4 Degenhardt L, Grebely J, Stone J, et al. Global patterns of opioid use and dependence: harms to populations, interventions, and future action. Lancet 2019;394:1560-79. doi:10.1016/S01406736(19)32229-9

5 Larney S, Peacock A, Mathers BM, Hickman M, Degenhardt L. A systematic review of injecting-related injury and disease among people who inject drugs. Drug Alcohol Depend 2017;171:39-49. doi:10.1016/j.drugalcdep.2016.11.029

6 Bradley H, Rosenthal EM, Barranco MA, Udo T, Sullivan PS, Rosenberg ES. Use of Population-Based Surveys for Estimating the Population Size of Persons Who Inject Drugs in the United States. I Infect Dis 2020;222(Suppl 5):S218-29. doi:10.1093/infdis/jiaa318

7 Werb D, Buxton J, Shoveller J, Richardson C, Rowell G, Wood E. Interventions to prevent the initiation of injection drug use: a systematic review. Drug Alcohol Depend 2013;133:669-76. doi:10.1016/j.drugalcdep.2013.08.017

8 Knaul FM, Farmer PE, Krakauer EL, et al, Lancet Commission on Palliative Care and Pain Relief Study Group. Alleviating the access abyss in palliative care and pain relief-an imperative of universal health coverage: the Lancet Commission report. Lancet 2018;391:1391-454. doi:10.1016/S01406736(17)32513-8

9 Busse JW, Wang L, Kamaleldin M, et al. Opioids for Chronic Noncance Pain: A Systematic Review and Meta-analysis. JAMA 2018;320:244860. doi:10.1001/jama.2018.18472

10 Chou R, Turner JA, Devine EB, et al. The effectiveness and risks of long-term opioid therapy for chronic pain: a systematic review for a National Institutes of Health Pathways to Prevention Workshop. Ann Intern Med 2015:162:276-86. doi:10.7326/M14-2559

11 Vowles KE, McEntee ML, Julnes PS, Frohe T, Ney JP, van der Goes DN. Rates of opioid misuse, abuse, and addiction in chronic pain: a systematic review and data synthesis. Pain 2015;156:569-76. doi:10.1097/01.j.pain.0000460357.01998.f

12 Busse JW, Craigie S, Juurlink DN, et al. Guideline for opioid therapy and chronic noncancer pain. CMA/ 2017;189:E659-66. doi:10.1503/cmaj.170363

13 Dowell D, Haegerich TM, Chou R. CDC Guideline for Prescribing Opioids for Chronic Pain - United States, 2016. MMWR Recomm Rep 2016;65:1-49. doi:10.15585/mmwr.rr6501e1

14 Lynch ME. The need for a Canadian pain strategy. Pain Res Manag 2011;16:77-80. doi:10.1155/2011/654651

15 King NB, Fraser V, Boikos C, Richardson R, Harper S. Determinants of increased opioid-related mortality in the United States and Canada, 1990-2013: a systematic review. Am J Public Health 2014;104:e32 42. doi:10.2105/AJPH.2014.301966

16 DeWeerdt S. Tracing the US opioid crisis to its roots. Nature 2019;573:S10-2. doi:10.1038/d41586-019-02686-2

17 Campbell G, Nielsen S, Bruno R, et al. The Pain and Opioids IN Treatment study: characteristics of a cohort using opioids to manage chronic non-cancer pain. Pain 2015;156:231-42. doi:10.1097/01.j.pain.0000460303.63948.8e

18 Wilton J, Chong M, Abdia Y, et al. Cohort profile: development and characteristics of a retrospective cohort of individuals dispensed prescription opioids for non-cancer pain in British Columbia, Canada. BMJ Open 2021;11:e043586. doi:10.1136/ bmjopen-2020-043586

19 Ray GT, Bahorik AL, VanVeldhuisen PC, Weisner CM, Rubinstein AL, Campbell $\mathrm{Cl}$. Prescription opioid registry protocol in an integrated health system. Am J Manag Care 2017;23:e146-55.

20 Compton WM, Jones CM, Baldwin GT. Relationship between Nonmedical Prescription-Opioid Use and Heroin Use. N Engl) Med 2016;374:154-63. doi:10.1056/NEJMra1508490

21 Mars SG, Bourgois P, Karandinos G, Montero F, Ciccarone D. “Every 'never' I ever said came true": transitions from opioid pills to heroin injecting. Int J Drug Policy 2014;25:257-66. doi:10.1016/j. drugpo.2013.10.004

22 Lankenau SE, Teti M, Silva K, Jackson Bloom J, Harocopos A, Treese $\mathrm{M}$. Initiation into prescription opioid misuse amongst young injection drug users. Int J Drug Policy 2012;23:37-44. doi:10.1016/j. drugpo.2011.05.014

23 Nolte K, Drew AL, Friedmann PD, Romo E, Kinney LM, Stopka TJ. Opioid initiation and injection transition in rural northern New England: A mixed-methods approach. Drug Alcohol Depend 2020;217:108256. doi:10.1016/j. drugalcdep.2020.108256

24 Syvertsen JL, Paquette CE, Pollini RA. Down in the valley: Trajectories of injection initiation among young injectors in California's Central Valley. Int J Drug Policy 2017;44:41-9. doi:10.1016/j. drugpo.2017.03.003

25 Cicero TJ, Ellis MS. Oral and non-oral routes of administration among prescription opioid users: Pathways, decision-making and directionality. Addict Behav 2018;86:11-6. doi:10.1016/j. addbeh.2018.05.015

26 Darnall BD, Juurlink D, Kerns RD, et al. International Stakeholder Community of Pain Experts and Leaders Call for an Urgent Action on Forced Opioid Tapering. Pain Med 2019;20:429-33. doi:10.1093/ pm/pny228

27 Oliva EM, Bowe T, Manhapra A, et al. Associations between stopping prescriptions for opioids, length of opioid treatment, and overdose or suicide deaths in US veterans: observational evaluation. BMJ 2020;368:m283. doi:10.1136/bmj.m283

28 Mark TL, Parish W. Opioid medication discontinuation and risk of adverse opioid-related health care events. J Subst Abuse Treat 2019;103:58-63. doi:10.1016/j.jsat.2019.05.001

29 Lynch M. Nonmedical use of prescription opioids: what is the real problem?Pain Res Manag 2013;18:67-8. doi:10.1155/2013/824914

30 Janjua NZ, Islam N, Kuo M, et al, BC Hepatitis Testers Cohort Team. Identifying injection drug use and estimating population size of people who inject drugs using healthcare administrative datasets. Int I Drug Policy 2018;55:31-9. doi:10.1016/j.drugpo.2018.02.001

31 McGrew KM, Homco JB, Garwe T, et al. Validity of International Classification of Diseases codes in identifying illicit drug use target conditions using medical record data as a reference standard: A systematic review. Drug Alcohol Depend 2020;208:107825. doi:10.1016/j.drugalcdep.2019.107825

32 Janjua NZ, Kuo M, Chong M, et al. Assessing Hepatitis C Burden and Treatment Effectiveness through the British Columbia Hepatitis Testers Cohort (BC-HTC): Design and Characteristics of Linked and Unlinked Participants. PLoS One 2016;11:e0150176. doi:10.1371/ journal.pone.0150176

33 Von Korff M, Saunders K, Thomas Ray G, et al. De facto long-term opioid therapy for noncancer pain. Clin J Pain 2008;24:521-7. doi:10.1097/AJP.0b013e318169d03b

34 Smolina K, Crabtree A, Chong M, et al. Patterns and history of prescription drug use among opioid-related drug overdose cases in British Columbia, Canada, 2015-2016. Drug Alcohol Depend 2019;194:151-8. doi:10.1016/j.drugalcdep.2018.09.019

35 Mamdani M, Rochon PA, Juurlink DN, et al. Observational study of upper gastrointestinal haemorrhage in elderly patients given selective cyclo-oxygenase-2 inhibitors or conventional non-steroidal anti-inflammatory drugs. BMJ 2002;325:624. doi:10.1136/ bmj.325.7365.624

36 Austin PC, Stuart EA. Moving towards best practice when using inverse probability of treatment weighting (IPTW) using the propensity score to estimate causal treatment effects in observational studies. Stat Med 2015;34:3661-79. doi:10.1002/ $\operatorname{sim} .6607$

37 Lee EW, Wei LJ, Amato DA, Leurgans S. Cox-Type Regression Analysis for Large Numbers of Small Groups of Correlated Failure Time Observations. Survival Analysis: State of the Art. Springer Netherlands, 1992: 237-47.

38 Lin DY, Wei LJ, Ying Z. Checking the Cox model with cumulative sums of martingale-based residuals. Biometrika 1993;80:557-72. doi:10.1093/biomet/80.3.557.

39 Fine JP, Gray RJ. A Proportional Hazards Model for the Subdistribution of a Competing Risk. J Am Stat Assoc 1999;94:496-509. doi:10.108 0/01621459.1999.10474144.

40 Rimawi M, Hilsenbeck SG. Making sense of clinical trial data: is inverse probability of censoring weighted analysis the answer to crossover bias?/ Clin Oncol 2012;30:453-8. doi:10.1200/ ICO.2010.34.2808

41 Tian TY, Zlateva I, Anderson DR. Using electronic health records data to identify patients with chronic pain in a primary care setting. I Am Med Inform Assoc 2013;20(e2):e275-80. doi:10.1136 amiajnl-2013-001856

42 Tonelli M, Wiebe N, Fortin M, et al, Alberta Kidney Disease Network. Methods for identifying 30 chronic conditions: application to administrative data. BMC Med Inform Decis Mak 2015;15:31. doi:10.1186/s12911-015-0155-5

43 Behar E, Bagnulo R, Knight K, Santos G-M, Coffin PO. “Chasing the pain relief, not the high": Experiences managing pain after opioid reductions among patients with HIV and a history of substance use. PLoS One 2020;15:e0230408. doi:10.1371/journal.pone.0230408

44 Voon P, Callon C, Nguyen P, et al. Denial of prescription analgesia among people who inject drugs in a Canadian setting. Drug Alcohol $\operatorname{Rev} 2015 ; 34: 221-8$. doi:10.1111/dar.12226 
45 Canadian Pain Task Force. Chronic Pain in Canada: Laying a Foundation for Action. 2019. https://www.canada.ca/content/ dam/hc-sc/documents/corporate/about-health-canada/public engagement/external-advisory-bodies/canadian-pain-task-force/ report-2019/canadian-pain-task-force-June-2019-report-en.pdf

46 Skelly AC, Chou R, Dettori JR, et al. Noninvasive Nonpharmacological Treatment for Chronic Pain: A Systematic Review Update. Agency for Healthcare Research and Quality (US). 2020. doi:10.23970/ AHROEPCCER227.

47 Hong T, Wang H, Li G, Yao P, Ding Y. Systematic Review and Meta-Analysis of 12 Randomized Controlled Trials Evaluating the Efficacy of Invasive Radiofrequency Treatment for Knee Pain and Function. Biomed Res Int 2019;2019:9037510. doi:10.1155/2019/9037510

48 Muhuri P, Gfroerer M, Davies C. Associations of Nonmedical Pain Reliever Use and Initiation of Heroin Use in the United States. 2013. https://www.samhsa.gov/data/sites/default/files/DR006/DR006/ nonmedical-pain-reliever-use-2013.htm

49 Cerdá M, Santaella J, Marshall BDL, Kim JH, Martins SS. Nonmedical Prescription Opioid Use in Childhood and Early Adolescence Predicts Transitions to Heroin Use in Young Adulthood: A National Study. J Pediatr 2015;167:605-12.e1, 2. doi:10.1016/j.jpeds.2015.04.07
50 Banerjee G, Edelman EJ, Barry DT, et al. High-dose prescribed opioids are associated with increased risk of heroin use among United States military veterans. Pain 2019;160:2126-35. doi:10.1097/j. pain.0000000000001606

51 Banerjee G, Edelman EJ, Barry DT, et al. Non-medical use of prescription opioids is associated with heroin initiation among US veterans: a prospective cohort study. Addiction 2016;111:2021-31. doi:10.1111/add.13491

52 Carlson RG Nahhas RW, Martins SS, Daniulaityte R. Predictors of transition to heroin use among initially non-opioid dependent illicit pharmaceutical opioid users: A natural history study. Drug Alcohol Depend 2016;160:127-34. doi:10.1016/i.drugalcdep.2015.12.026

53 Surratt HL, Kurtz SP, Buttram M, Levi-Minzi MA, Pagano ME, Cicero T]. Heroin use onset among nonmedical prescription opioid users in the club scene. Drug Alcohol Depend 2017;179:131-8. doi:10.1016/j. drugalcdep.2017.06.034

54 Kelley-Quon LI, Cho J, Strong DR, et al. Association of Nonmedical Prescription Opioid Use With Subsequent Heroin Use Initiation in Adolescents. JAMA Pediatr 2019;173:e191750. doi:10.1001/ jamapediatrics.2019.1750

Web appendix: Supplementary Appendix 